\title{
A High Efficiency, Miniaturized Ka Band Traveling Wave Tube Based on a Novel Finned Ladder RF Circuit Design
}

\author{
E.G. Wintucky, J.D. Wilson, K.R. Vaden, D.A. Force, J.C. Freeman, G.G. Lesny \\ NASA Glenn Research Center, 21000 Brookpark Rd., Cleveland, OH 44135 \\ Email: Edwin.G.Wintucky@grc.nasa.gov),Tel: 216-433-3510 \\ C.L. Kory, C.T. Chevalier \\ Analex Corporation, 3001 Aerospace Parkway, Brook Park, OH 44142 \\ Email: Carol.L.Kory@grc.nasa.gov, Tel: 216-433-3512 \\ B. Ebihara \\ Ohio Aerospace Institute, 22800 Cedar Point Rd., Brook Park, OH 44142 \\ Email: benebihara@oai.org, Tel: 440-962-3133 \\ J.A. Dayton \\ Consultant \\ Email: jd10423@aol.com, Tel: 216-961-1696
}

Space communications architectures are being planned to meet the high rate data distribution requirements of future NASA Enterprise missions. These will require the use of traveling wave tube amplifiers (TWTAs) to provide the high frequency, RF power and efficiency needed for many of the communications links. Future missions will also employ smaller spacecraft with corresponding requirements of reduced size and weight of the onboard communications systems. A program addressing these requirements is currently underway at NASA Glenn Research Center (GRC)* for the development of a high efficiency, 20 watt, $32 \mathrm{GHz}$ TWT of reduced size and weight that is based on a novel high gain slow-wave circuit design, termed the "finned ladder". The goal is $60 \%$ overall efficiency with efficiency enhancement resulting from computational optimization of the RF circuit and multistage depressed collector designs and the use of an electrically efficient cathode. Although a frequency of $32 \mathrm{GHz}$ and a moderate $\mathrm{RF}$ power of $20 \mathrm{~W}$ have been selected for development, the TWT can be readily scaled for operation over a broad range of frequencies and power levels.

The finned ladder slow-wave circuit, designed using the computer programs MAFIA, Microwave Studio (MWS) and GRC developed advanced optimization software, has an exceptionally high gain per unit length. For operation at $6.8 \mathrm{kV}$ and $32 \mathrm{GHz}$, the period is on the order of $0.5 \mathrm{~mm}$. A section of the RF circuit is shown in Figure 1. The very high $R F /$ electron beam interaction impedance $(>100 \mathrm{ohms})$ enables more than $40 \mathrm{~dB}$ of gain over a $5 \mathrm{~cm}$ length and an RF efficiency greater than $20 \%$, which offers a significant reduction in RF circuit length and the potential for TWT miniaturization. As shown in the computer simulated (MAFIA) mode diagram in Figure 2, only the fundamental forward wave mode is excited, indicating the absence of instability due to the backward wave present in helical circuits. Cold test results show excellent agreement with the computer simulated (MWS) dispersion curve (Figure 3).

One of the approaches being pursued for RF circuit fabrication is the stacking and bonding of the period disk elements (alternating the active circuit elements containing the beam tunnel with spacer elements). The unusual geometry, small dimensions (as small as

This is a preprint or reprint of a paper intended for presentation at a conference. Because changes may be made before formal publication, this is made available with the understanding that it will 
$0.1 \mathrm{~mm}$ ) and close tolerances (as small as 2.5 microns) of the RF circuit, require the use of microfabrication methods. Among the methods under investigation are photochemical machining, high precision laser machining and high precision Electric Discharge Machining (EDM).

Details on the finned ladder TWT design will be presented, as well as progress on the fabrication of the RF circuit.
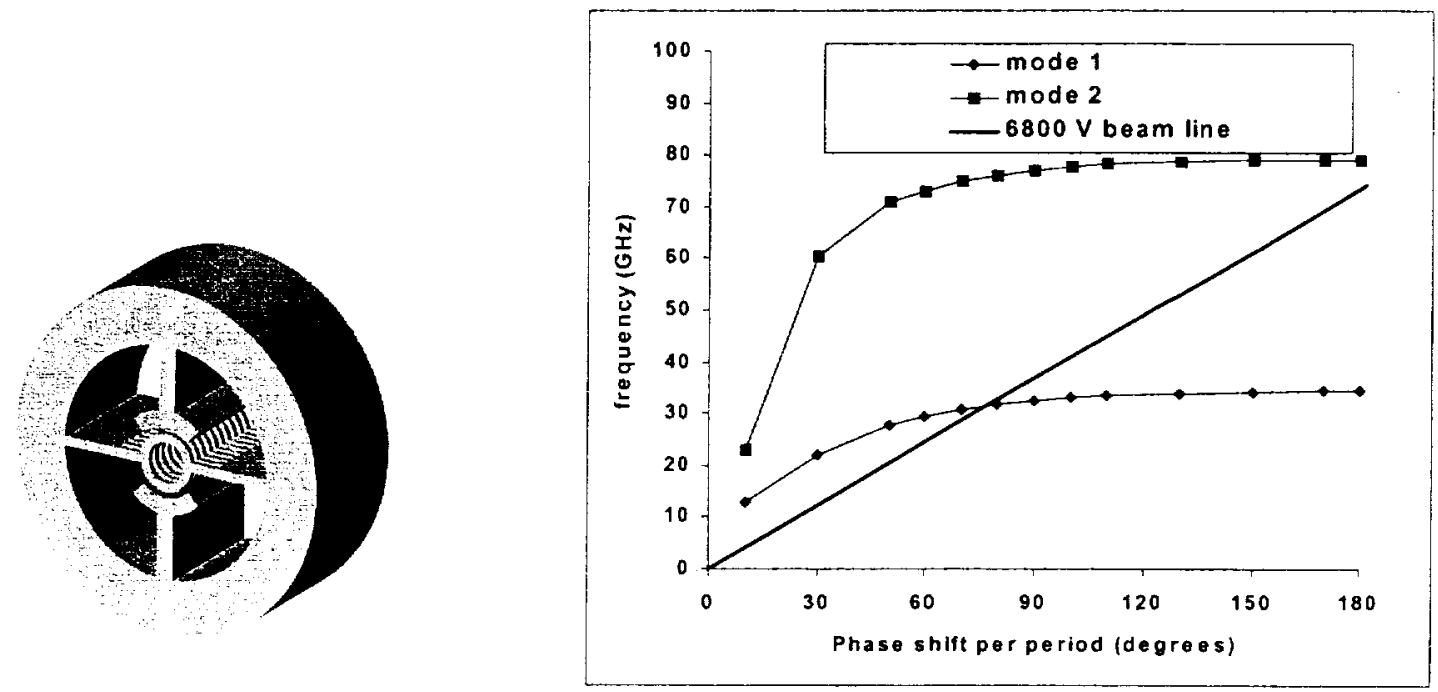

Figure 1. 3D view of section of "finned ladder" periodic RF circuit

Figure 2. Computer simulated mode diagram (MAFIA)

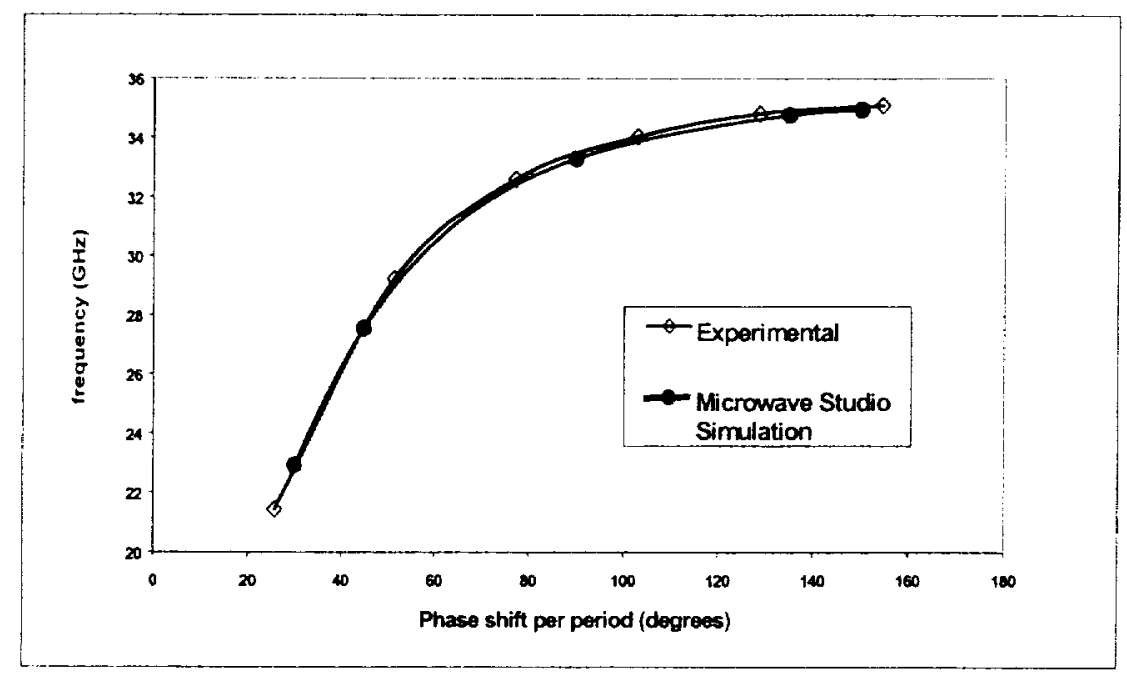

Figure 3. Simulated (MWS) and experimental dispersion curves

*This work is supported by the NASA Computation, Information and Communications Technology (CICT) program (Code R) 